\title{
EXPLORING THE BOMBARDMENT HISTORY OF THE MOON
}

\author{
Community White Paper to the \\ Planetary Science and Astrobiology Decadal Survey \\ 2023-2032
}

\section{Sanctioned by the NASA Lunar Exploration Analysis Group}

July 15, 2020

\author{
Author: \\ William F. Bottke
}

\section{Endorsers:}

\begin{tabular}{ll}
\hline Endorser & Affiliation \\
\hline Joseph M. Boyce & University of Hawaii \\
Ian Crawford & Birkbeck College, University of London, UK \\
Qing-Zhu Yin & University of California at Davis, Department of \\
& Earth and Planetary Sciences \\
Kip Hodges & Arizona State University \\
Caitlin Ahrens & University of Arkansas \\
Stephen Elardo & Department of Geological Sciences, University of \\
& Florida \\
Kris Zacny & Honeybee Robotics \\
Daoru Han & Missouri University of Science and Technology \\
Marc Norman & Australian National University \\
Gordon Osinski & University of Western Ontario \\
Amy Mainzer & University of Arizona \\
J. R. Szalay & Princeton University \\
Shashwat Shukla & University of Twente, The Netherlands \\
Jonti Horner & University of Southern Queensland \\
Audrey Bouvier & Universität Bayreuth
\end{tabular}


Katherine Joy

Ross W. K. Potter

Barbara Cohen

Heather Meyer

Luke Dones

Timothy D. Swindle

Kirby D. Runyon

Ross A. Beyer

Stephen Mojzsis

Simone Marchi

Dimitri A. Papanastassiou

Christian Tai Udovicic

Nicolle Zellner

Michelle Kirchoff

Meng-Hua Zhu

Amy J. V. Riches

Csilla Orgel

Jaclyn D. Clark

Carle M. Pieters

James W. Head

Maria E. Banks

Vincent F Chevrier

Kim Cone

Katherine Shirley

Jennifer Lopez

Benjamin Greenhagen

Gwendolyn Bart

Christian Koeberl

Rebecca Ghent

Melissa Roth

Vince Roux
The University of Manchester, UK

Brown University

NASA Goddard Space Flight Center

JHU APL

Southwest Research Institute

University of Arizona

Johns Hopkins APL

SETI Institute and NASA Ames Research Center

University of Colorado at Boulder

SwRI

Geol. Planet. Sci., Caltech

Northern Arizona University

Albion College

Southwest Research Institute

Macau University of Science and Technology

UK

ESA-ESTEC

Arizona State University

Brown University

Brown University

NASA Goddard Space Flight Center

University of Arkansas

Colorado School of Mines

University of Oxford

Astrobotic

Johns Hopkins Applied Physics Laboratory

University of Idaho

University of Vienna

Planetary Science Institute

Off Planet Research, LLC

Off Planet Research, LLC 


\section{EXECUTIVE SUMMARY}

The Moon is the only object in the Solar System that is both relatively accessible and still bears evidence, in the form of craters, shocked materials, and regolith, from practically every epoch of Solar System history. This is both a challenge and a blessing. It is challenging because to understand the Moon's internal structure, chemistry, and complex resurfacing and bombardment history, we need to understand the formation and evolution of the Solar System as a whole. It is a blessing because the Moon is a treasure trove of information for the study of the events that have shaped the Earth and other planets during most of their histories. To understand the Moon, we must study the planets, and to understand the planets, we must study the Moon.

The critical importance of interpreting the bombardment history of the Moon, and how it can be used to better understand key issues such as planet formation and migration, has been emphasized in different reports from authoritative committees. For example:

- The National Research Council's 2007 Space Studies Board report, "The Scientific Context for the Exploration of the Moon" made it their top science goal.

- The update of this report, namely the 2018 Lunar Exploration Analysis Group's report “Advancing the Science of the Moon: Report of the Specific Action Team” (ASM-SAT report) made it their top priority science concept (out of eight concepts).

- The primary questions of the 2010 Decadal Survey's theme "Building New Worlds" can be addressed by understanding the Moon's bombardment history (e.g., it can constrain both the timing and nature of terrestrial planet formation and giant planet migration).

0 What were the initial stages, conditions, and processes of Solar System formation and the nature of the interstellar matter that was incorporated?

o How did the giant planets and their satellite systems accrete, and is there evidence that they migrated to new orbital positions?

o What governed the accretion, supply of water, chemistry, and internal differentiation of the inner planets and the evolution of their atmospheres, and what roles did bombardment by large projectiles play?

The Moon's bombardment history is also uniquely intertwined with that of Earth. While terrestrial impact events have affected the origin and evolution of our atmosphere, environment, and the prospects for habitability/early life, the impact record itself on Earth > 650 Ma has been almost entirely erased. Accordingly, the timing and nature of impacts on the Moon allow us to infer how related impacts have affected the evolution of Earth (and astrobiology!).

In addition, links between crater spatial densities and the radiometric ages of lunar samples serve as the basis for estimating surface ages for other solid bodies (e.g., Mars). The problem is that lunar chronology is (i) based on limited data for $<3.2 \mathrm{Ga}$ and $>3.9 \mathrm{Ga}$ terrains and (ii) is applied to ancient surfaces on other worlds using questionable assumptions (e.g., the unknown impact flux on Mars and Moon > 3.9 $\mathrm{Ga}$ ). This means our interpretation of data from important surfaces, such as the rover landing sites on Mars, may be based on flawed assumptions. This makes it imperative to understand the production rate of lunar craters over its entire history.

Finally, understanding the impact flux history recorded on the Moon gives us an idea of whether future catastrophic impacts are likely to affect the Earth or, on a different scale, how impacts from small particles should affect the planning for human space-flight missions.

This white paper will emphasize the top science goals related to the Moon's 


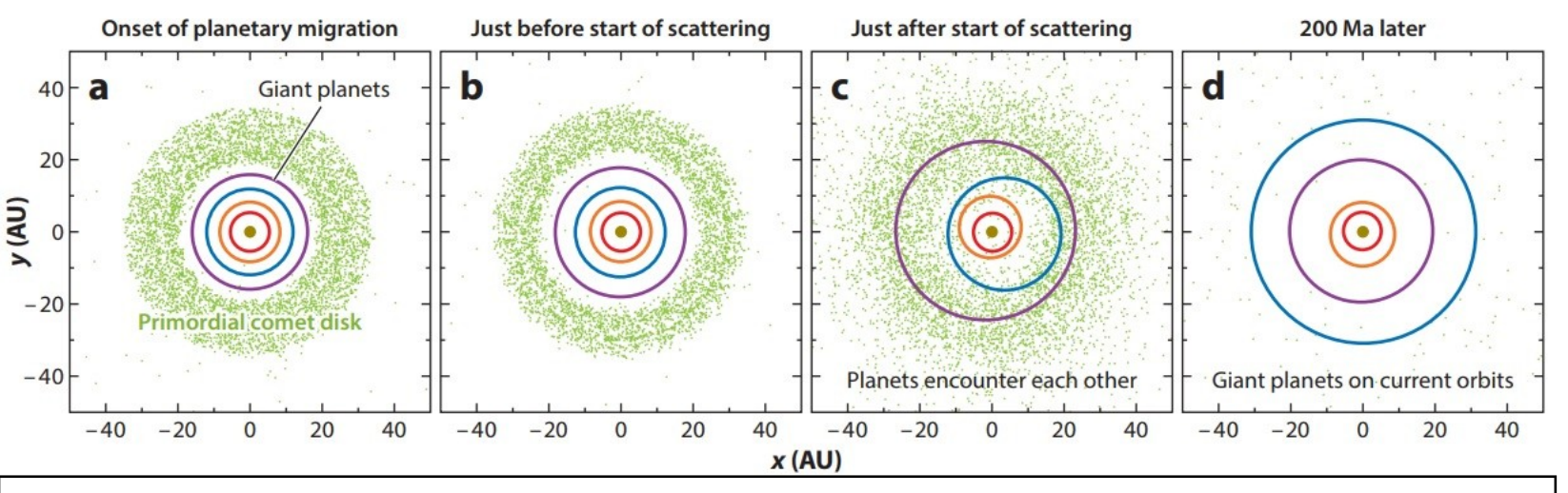

Fig. 1. Giant planet migration and dynamical instability. The giant planets scatter each other, while Neptune migrates into a massive outer disk of cometesimals (i.e. primordial comets). This migration dynamically affects the terrestrial planets/asteroid belt (e.g,. Nesvorný 2018).

bombardment history within the ASM-SAT report. Exploring them could produce transformational science from future space exploration.

\section{SCIENCE GOALS}

The top science concept of the ASM-SAT report, and that of 2007 NRC SCEM report, is "The bombardment history of the inner Solar System is uniquely revealed on the Moon. We focus our discussion on the key science goals coming from this concept.

\section{ASM-SAT Science Goal 1a-Test the cataclysm hypothesis by determining the spacing in time of the creation of lunar basins.}

The story of early lunar bombardment cannot be told without an understanding of planet formation and giant planet migration. We refer the reader to the SBAG-authorized white paper, “The Evolution of Small Body Populations: From Planet Migration to Thermal Drift Forces" by Bottke et al., which complements the discussion below. Here we summarize some of that material.

There is now broad agreement that the giant planets migrated over large distances early in their history, based on their orbital eccentricities and inclinations, the structure of the Kuiper Belt, the orbit and size distributions of comet-like bodies within the asteroid belt, Trojans, irregular satellites, and so on (see reviews and references in [1, 2]). Models suggest that after the solar nebula dispersed, our Solar System was in a different configuration than the one seen today. Gas accretion left the giant planets on nearly circular coplanar orbits between 5 and 20 au, with most/all locked in mutual mean motion resonances with one another. The primordial Kuiper belt existed beyond the original orbit of Neptune, with an approximate population of 20 Earth masses in small comet-like bodies that stretched from 20 au to the cold classical Kuiper belt.

This configuration, however, eventually went unstable [3]. The explanation for the dynamical structure of the present-day Kuiper Belt is that the outermost giant planets radially migrated through the primordial comet disk in a violent exchange of orbital energy and angular momentum. This led to a reorganization of the giant planets in what is now called a "giant planet instability" [1]. As the giant planets moved to their current orbits, stable reservoirs of materials became destabilized, causing enhanced bombardment rates throughout the Solar System [e.g., 4].

Our thinking about the timing of giant planet migration, however, has dramatically changed over the last few years. Late migration models, in which a delay between planet formation and migration produced a discrete "cataclysm", or "late heavy bombardment" (LHB) episode 500 Myr after the Earth-Moon system formed, now appear to violate key constraints (e.g., [5]). New work instead argues for early migration ( $<100$ Myr after CAIs). If so, the giant planet instability affected terrestrial planet formation in a profound manner.

The sources and timing of early lunar bombardment have been debated for decades, largely because constraints have been limited (e.g., [4]). At present, there are three likely sources of early impactors for the inner Solar System:

- Comets, defined as icy bodies from the outer Solar System beyond Neptune, 
- Leftover planetesimals, defined as remnants of terrestrial planet formation $(<1.5 \mathrm{au})$, and

- Asteroids, defined as rocky bodies that formed or were captured between 2 to 5 au.

The timing of when these different populations hit the Moon en masse is unknown. Until recently, it was thought that the dominant source of early impactors on Moon/Mars were leftover planetesimals, while comets/asteroids struck $\sim 4 \mathrm{Ga}$ as a byproduct of late giant planet migration. If giant planet migration occurred during the terrestrial planet formation era, however, all three populations were hitting the Moon -- and other inner Solar System worlds -- simultaneously during their developmental phases [5, 6].

The timing of the giant planet instability is unknown. It is possible it took place shortly after the dissipation of the Solar nebula 3-10 Myr after the birth of the Sun; [7, 8]. If so, many small bodies destabilized from their source reservoirs may have roamed the Solar System well before the formation of the Moon, which arguably took place 40-60 Myr after the formation of the first solids [e.g., 9]. The ancient surfaces of the Moon might only record the tail end of this bombardment. On the other hand, if the instability occurred many tens of Myr after CAIs, the mix of impactors striking the early Moon (and Mars/Mercury) could have been very different.

A complication concerning the interpretation of the Moon's bombardment record is that large lunar impact basins might not be preserved by a magma ocean or by a slushy, low viscosity crust [e.g., 6, 10]. The timing of when this takes place for the Moon's nearside and farside surfaces, whose crustal thicknesses are very different [11,12], has yet to be determined. A similar problem could affect ancient surfaces like Mercury and Mars [e.g., 13]. This could mean that the peak of early lunar bombardment may not have an obvious surface expression on the Moon, or that the most ancient surfaces of the Moon, Mars, and Mercury show different bombardment expressions representing the times when their surfaces became stable.

In summary, when evaluating the signatures of early bombardment, one must consider (i) the starting time of the giant planet instability, (ii) the bombardment profile of each impactor population, and (iii) the retention times of impact structures on the Moon (and other worlds).

Where does this leave the cataclysm? Since the time of the Apollo Moon landings 50 years ago, there has been intense debate in the literature concerning the nature of the lunar impact record from 4.5-3.8 billion years ago (Ga) [4]. The cataclysm was originally defined as the time period $\sim 3.7$ to $\sim 3.9$ Ga when numerous $D>300 \mathrm{~km}$ diameter craters, known as basins, formed on the Moon. The question is whether the cataclysm is real or an artifact of sampling biases.

From a dynamical standpoint, if we assume early giant planet migration determines the endgame of terrestrial planet formation, impactor populations, whether asteroids, comets, or leftover planetesimals, should yield “declining bombardments”, defined as lots of early impacts and few late ones (e.g., [14, 15]). Presumably, this would rule out a cataclysm scenario. The problem is that most impactor populations dynamically decay away relatively quickly, and that should leave the Moon with many more early basins than are observed [e.g., 6]. One potential way out of this dilemma is to assume that early basins formed prior to $4.3 \mathrm{Ga}$ on the Moon, Mars, etc. were completely erased [e.g., 6].

Curiously, two of the three largest lunar basins, Imbrium and Orientale ( $900-1100 \mathrm{~km}$ diameter), formed in the cataclysm era [4]. Serenitatis and Crisium, which are only modestly smaller basins, have approximately the same spatial densities of GRAIL-detected $D>90 \mathrm{~km}$ craters as Imbrium [16]. Accordingly, if Imbrium is 3.9 Ga, Orientale, Serenitatis, and Crisium should probably have comparable ages. The formation of all these large basins in the cataclysm era is hard to reconcile with a simple declining bombardment, and it could argue that something else was going on. Testing the cataclysm hypothesis, however, will require new data; other basin ages cited in the older literature, such as those for Nectaris, cannot be supported [4]. 
ASM-SAT Science Goal 1b-Anchor early Earth-Moon impact flux curve by determining the age of (perhaps) the oldest and largest lunar basin (South Pole-Aitken Basin).

The pre-Nectarian era on the Moon begins with the formation of the Moon, possibly $4.5 \mathrm{Ga}$, and ends with the formation of Nectaris basin [17]. In terms of bombardment, nearly all preNectarian basins and craters exist on the ancient lunar farside [4,16,17,18]. Unfortunately, it is impossible at this time to conclusively relate dated pre-Nectarian rock clasts to any specific preNectarian geologic surface unit because subsequent impact events have displaced these samples from their original geographic location after formation.

The oldest and largest (undisputed) basin on the Moon, as defined by the spatial densities of GRAIL-detected $D>90 \mathrm{~km}$ craters [16], is South Pole-Aitken basin (SPA). SPA is twice the diameter of Imbrium and is comparable in size to the Hellas and Utopia basins on Mars. As the oldest impact basins are critical calibration points for lunar chronology, finding the age of SPA or any pre-Nectarian feature has implications for other planetary surfaces whose inferred surface ages are linked to lunar data via crater counts. Therefore, it is of critical importance to date the oldest generally recognized impact basin in order to derive the beginning of the Moon's bombardment history. Moreover, in this context, it is useful to recall that the Moon faithfully records events during Earth's Hadean Eon that relate directly to the period when the molecular precursors to life emerged [4].

Some implications for SPA formation at different times are as follows:

- SPA forms shortly after the Moon's formation. SPA may have been made by comets, asteroids, or leftover planetesimals [5,6,19]. It would also argue there was little erasure of early lunar basins, there were relatively few large impactors, and that the impactor population itself had to dynamically decay away very slowly to explain observations.

- $\quad$ SPA forms 4.3 Ga. SPA was probably made by leftover planetesimals, whose impactors may have a slower dynamical decay profile than comets or asteroids [5,14,19]. It would suggest most early lunar basins were in fact erased, and that recorded basins on the Moon, Mars, and Mercury was predominantly made late by the tail of a single impactor population.

- SPA forms 4.0 Ga. This would argue that the cataclysm scenario is viable and that current models of planet formation/migration/evolution are missing something [4].

Note that if one could date all the lunar basins that ever formed, it might be possible to weigh the contributions of different impactor populations. Those that formed from leftover planetesimals could be particularly interesting because any surviving impactor fragments and/or isotopic signatures might tell us about the unevolved progenitor material that made the Earth and other terrestrial planets.

Thus, the Moon potentially allows us to understand the last stages of planet formation throughout the Solar System. To test this tantalizing hypothesis, however, we need to determine the magnitude and duration of the lunar bombardment. Dynamically, this means further probing planet formation processes to see how constraints from the Moon and other objects (e.g., Mars, asteroid belt, outer planet satellites) fit together within a unified model. Geochemically, this means collecting impact melts unambiguously tied to large craters and basins, and minerals thermally affected by impacts, for detailed petrologic, geochemical, and geochronological analyses. These samples should be selected to represent the entire distribution of stratigraphic ages among basin-forming events, and of lunar geographic locations. These same rocks and minerals also hold the potential to constrain the source of projectiles and their chemical compositions. 
An important lesson-learned from the Apollo era concerns the selection of multiple sample locations. The Apollo landing sites covered a relatively small region of the lunar surface $[4,17]$. An ongoing controversy has been whether or not samples which purport to date the cataclysm are substantially contaminated by a single local event, namely the formation of the Imbrium impact basin $[4,17]$. Ensuring spatial diversity of sample locations would potentially mitigate the development of similar future controversies.

We close this section by pointing out a few applications of this work that may allow us to address profound science questions beyond the Moon. Consider the following examples:

- Widely-adopted chronologies for Mars have the Early Noachian period extending to $4.1 \mathrm{Ga}$, the assumed age of the Hellas basin [e.g., see references in 13]. If many lunar basins formed $>4.3 \mathrm{Ga}$, however, that would likely be true for Mars as well. Thus, Martian Noachian history, and all of its activity in terms of flowing water/volcanism, might need to be pushed far back in time.

- The majority of Martian volatiles (e.g., water) could have been delivered by comets and/or hydrated asteroids during early bombardment [e.g., 5,6,20].

- The impact flux on the early Earth between 4.5 and $3.5 \mathrm{Ga}$, which is recorded on the Moon but has been erased by terrestrial geological processes, can be used to understand the impact regime under which life became established on our planet [e.g., 4].

\section{ASM-SAT Science Goal 1c_Establish a precise absolute chronology.}

After the basin-forming epoch of the first billion years, the Earth, Moon, and all other Solar System bodies continued to be targets of wayward asteroids and comets. The number of accumulated craters is directly related to the age of the surface [14]. Sometimes those impacts produced dramatic results, such as the geologically recent collision that excavated the Chicxulub impact crater and extinguished much life on Earth, including dinosaurs, 66 million years ago. Since the Earth's early geologic record has been largely erased, the Moon is the best preserved and most accessible place to constrain the ages of events that occur on planetary surfaces (chronology) by combining the frequency of craters accumulated over time with measured absolute sample ages.

However, the Apollo sites provide absolute ages for an extremely limited range of terrain on the lunar nearside [14]. At present a large discrepancy exists between impact flux models, with inferred ages differing by up to a billion years in the $3.5 \mathrm{Ga}$ to $2 \mathrm{Ga}$ time period [21]. Fluxes are also very poorly constrained for the last billion years due to lack of data. Removing such chronological discrepancies is key to understanding impact rates as life was evolving on Earth as well as when Mars was potentially habitable. Documenting the recent flux record on the Moon would provide important constraints for the present impact flux environment at $1 \mathrm{AU}$, of great interest for human civilization.

Determining the ages of samples from a suite of carefully selected presumed young lunar terrain and impact craters of varied sizes and stratigraphic ages would allow us to develop an accurate clock to be applied to planetary surfaces throughout the Solar System. Ages from 1 to 3 Ga terrains, e.g., near-side young basaltic terrains and large craters like Aristillus and Tycho, would address the time period during which available chronologies are highly uncertain. Accurate absolute ages would also enable testing of postulated impact flux spikes (e.g., 0.47, 0.8, 1.4 Ga, $2 \mathrm{Ga}$ ) that appear to be coincident with major changes in Earth’s biosphere [e.g., 22].

Additional science goals that affect our determination of lunar chronology include understanding the role of secondary craters on crater counts, latitudinal and apex/antapex 
asymmetries in crater counts; and crater formation as a process in itself (which allows us to compute scaling relationships between impactors and craters).

\section{ASM-SAT Science Goal 1d-Assess the recent impact flux.}

In this goal, "recent” depends on the science question asked; the last several Gy, which can help us understand the evolution of life on Earth, and the last several tens of My, which can help us probe more recent events and constrain the present-day hazard to human life from impacts.

Several lines of evidence indicate the majority of lunar impacts over the past 3 Gy have been by asteroids, not comets (e.g., [23]). Most Earth-impacting asteroids originate in the main asteroid belt, where they are affected by both collisions and dynamical processes. Models of the evolution of the asteroid belt have shown that the lunar impact rate must have been changing over time, with peaks and valleys controlled by the major catastrophic collisions and cratering events. Both kinds of events create numerous fragments that can be seen in today's asteroid belt as so-called "families" [24, 25, 26]. In fact, $>90 \%$ by number of main-belt asteroids with diameter $D>1 \mathrm{~km}$ are family members and so family members currently dominate the lunar impactor population. Hence, characterizing this dominant, stochastic component of the lunar impact record is crucial for any attempt to determine an accurate lunar chronology. The Moon can also help us determine whether asteroid disruption events are connected to impact-induced mass extinction events on Earth (e.g., "asteroid showers”) [24,25,26].

Even though comets have contributed only modestly to the Moon's overall cratering record over the last 3 billion years, they may still have been an important source of volatiles. Indeed, if near-surface ice or hydrated phyllosilicates are found in deep polar craters, a substantial fraction of the water may have come from such comets (or hydrated C/D/P-type asteroids). Gain or loss of volatiles in impacts depends on factors like the impact velocity, which can be determined by detailed dynamical calculations.

Finally, the flux of small particles striking the Moon is important to understanding lunar regolith processes as well as the nature of the hazard to humans and equipment on the surface of the Moon (or some other interesting world to be explored) [27]. The Moon is constantly being hit by tiny particles with high kinetic energies produced by both comets and asteroids. Meteor showers created by disrupted comets and asteroids also bombard the Moon with dust-sized particles at particular times. Unlike the Earth, however, these particles are not stopped by any atmosphere. This hazard must be dealt with if humans are to spend long intervals in space or at an outpost exposed to space.

\section{CONCLUSIONS}

The Moon is itself a fascinating object, but it can also be viewed as a "Rosetta Stone" for deciphering the histories of the other planets. The key is to understand lunar bombardment, the highest-priority lunar science goal as determined by the authoritative committees referenced in the Introduction. We believe the new Decadal Survey should closely examine what the Moon can do for the rest of the Solar System in their analysis. Moreover, it is essential that NASA continue to support $R \& A$ programs that allow progress to be made on these priorities.

The rationale for studying early lunar bombardment can be summarized

as follows. The history of impacts in the early Earth-Moon system, in particular around $3.9 \mathrm{Ga}$, the time that life was emerging on Earth, is a critical chapter in terrestrial planet evolution. Understanding early lunar bombardment is important for several reasons. 
It can help us:

- Test our models of terrestrial planet formation and giant planet migration.

- Determine the dominant environmental conditions at the Earth's surface when minimal terrestrial constraints exist, and whether impacts helped frustrate or encourage life.

- Constrain how the lunar magma ocean evolved and whether it affected the inferred chronology of not only the Moon but that deduced for many Solar System worlds.

- Answer the question of whether there was any kind of cataclysm at $\sim 3.9$ $\mathrm{Ga}$; this would likely require sample returns and/or in situ dating of a range of ancient impact basins.

- In a related manner, the age of South Pole-Aitken Basin is needed to help us understand when the record of early bombardment begins on the Moon and the type of impactor population that dominated the recorded impact flux. With that information, we can glean insights into issues such as the age of giant planet instability and the Moon itself.

It is also important the understand the impact record on the Moon over the last several billion years, and that means establishing a well-calibrated lunar chronology. This can be done by returning or dating samples from several key benchmark craters, young lava flows, and old impact basins. It can help us to:

- Find the ages of surfaces of other inner Solar System worlds through modeling.

- Determine when asteroid disruption events in the main asteroid belt produced surges in the inner Solar System impact flux. This information is critical to astrobiology studies, with strong impact surges potentially linked to changes in the Earth's biosphere. At present, limited information on the terrestrial impact record exists for times earlier than $650 \mathrm{Ma}$ [25].

\section{REFERENCES}

[1] Nesvorný, D. 2018. Ann. Res. Astron. Astrophys. 56, 137. [2] Morbidelli et al. 2015. In Asteroids IV. U. Arizona Press, 493-508. [3] Tsiganis, K., et al. 2005. Nature 435, 459. [4] Bottke, W.F and Norman, M. 2017. Ann. Res. Earth Planet Sci. 45, 619. [5] Nesvorný, D., et al. 2018. Nature Astron. 2, 878. [5] Clement, M. S., et al. 2018. Icarus 311, 340. [6] Morbidelli, A., et al. 2018. Icarus 305, 262. [7] Haisch, K. E., et al. 2001. Astrophys. J. 553, L153. [8] Masset, F. and Snellgrove, M. 2001. MNRAS 320, L55. [9] Barboni, M. et al. 2017. Sci. Adv. 3, 1. [10] Miljković, K., et al. 2013, Science 342, 724. [11] Wieczorek, M. A., et al. 2013. Science 339, 671. [12] Andrews-Hanna, J. C., et al. 2014. Nature 514, 68. [13] Bottke, W. F., AndrewsHanna, J., 2017. Nature Geosci. 10, 344. [14] Bottke, W. F. et al. 2007. Icarus 190, 203. [15] Brasser, R. et al. 2020. Icarus 338, 113514. [16] Evans, A. J., 2018. JGR-Planets 123, 1596. [17] Wilhelms, D. 1987. The Geologic History of the Moon, USGS Prof. Paper 1348. [18] Neumann, G. A. et al. 2015. Sci. Adv. 1, e1500852. [19] Nesvorný, D., et al. 2017. Astron. J. 153, 103. [20] Stern, A., \& Levison, H. 1999. LPSC 30, 1141. [21] Robbins, S. J., 2014. EPSL 403, 188. [22] Schmitz, B., et al. 2019, Sci. Adv., 5, eaax4184. [23] Bottke, W.F., et al. 2002. Icarus 156, 399. 
[24] Bottke, W. F. et al. 2007. Nature 449, 48. [25] Mazrouei, S., et al. 2019. Science 363, 253. [26] Nesvorny, D. et al. 2009. Icarus 200, 698. [27] Horányi, M. et al. Nature 522, 324. 